\title{
E-beam Nanolithographically Patterned Metal Oxide Nanowire Arrays for Highly Sensitive Gas Sensors
}

\author{
L. Campbell A. D., D. Smith, G. May, L. Caliste, Y. Chen, and W. L. Zhou \\ Advanced Materials Research Institute, University of New Orleans, LA 70148
}

New generation gas sensors based on metal oxide nanowires have shown increased sensitivity, smaller construction and greater versatility over the traditional thin film counterparts [1-5]. In this project, several techniques are integrated together to produce a highly sensitive compact gas sensor. The construction of the sensor can be broken down into three main sections: synthesis and characterization, architecture fabrication, and nanowire deposition.

First, metal oxide nanowires have been synthesized using a thermal evaporation method and characterized by Carl Zeiss 1530VP Field Emission Scanning Electron Microscopy (FESEM) and JEOL 2010 Transmission Electron Microscopy (TEM). Fig. 1 shows $\mathrm{ZnO}$ and SnO nanowires typical of those used in sensor fabrication. The size ranges from $50-200 \mathrm{~nm}$ in diameter.

Second, optical lithography is used to fabricate the larger architectures of the sensor, like the electrode pads and contacts. Then, e-beam nanolithography, integrated with the Carl Zeiss 1530VP FESEM, is used to fabricate the smaller architectures, such as the finger nanoelectrodes. E-bean nanolithography will also be employed to fabricate protective photoresist windows, which leaves the finger electrodes exposed during nanowire assembly. Fig.2 shows the progressions of architecture fabrication.

Lastly, a single, dense layer of nanowires will be deposited using the Langmuir-Blodgett technique. After deposition, the protective window is removed and the nanowires remaining on the finger nanoelectrodes compose the sensing area. Fig. 3 shows the deposition procedure. The sensor can then be tested to measure the electrical transport properties in the presence of different gasses in different concentrations. The detail will be discussed in the presentation.

\section{References}

[1] N. Barsan, M. Schweizer-Berberich, and W. Gëpel, Fresenius J. Anal.365, (1999) 287.

[2] W. Gëpel, Sens. Actuators A 56, (1996) 83

[3] M. Ferroni, V. Guidi, G. Martinelli, E. Comini, G. Sberveglieri, D. Boscarino, and G. Della Mea, J. Appl. Phys.88, (2000), 1097.

[4] E. Comini, G. Faglia, G. Sberveglieri, Z. Pan, and Z.L. Wang, Appl. Phys. Lett. 81, (2003) 1869.

[5] C. Li, D. Zhang, X. Liu, S. Han, J. Han, and C. Zhou, Appl. Phys. Lett. 82, (2003) 1613.

[6] We gratefully acknowledge the support of this work by the Advanced Materials Research Institute through the DARPA Grant No. MDA972-04-1-0029, National Science Foundation (EPS-0092001) and Louisiana Board of Regents (NSF/LEQSF(2001-04)-RII03). We thank CAMD of Louisiana State University for the use of clean room and photolithography facility. 


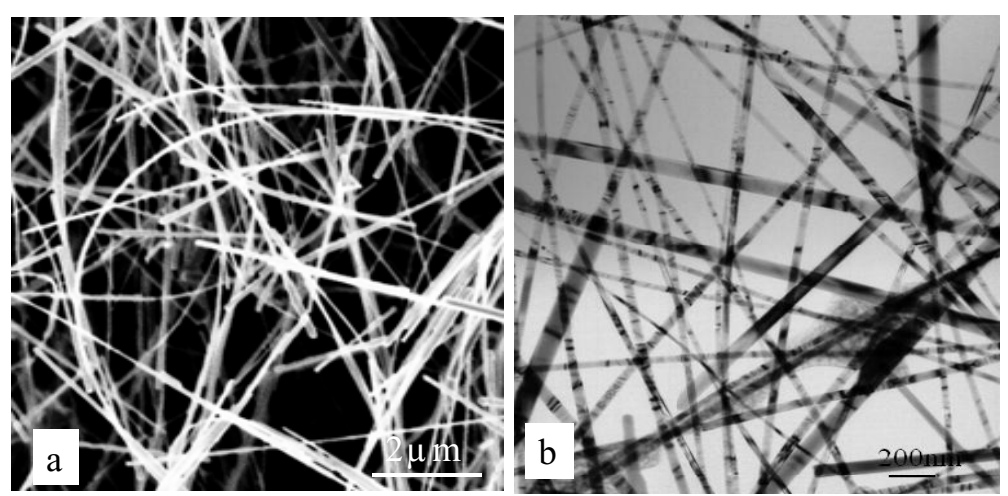

Fig. 1 (a) $\mathrm{ZnO}$ nanowires characterized by FESEM, (b) $\mathrm{SnO}$ characterized by TEM. These wires are typical of the wires used for sensor fabrication.
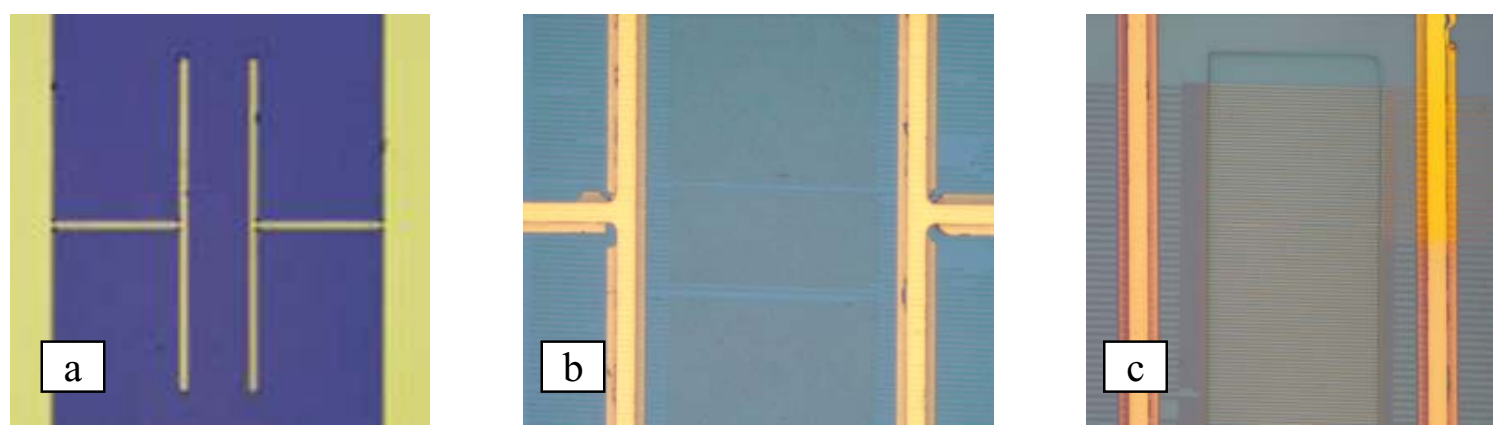

Fig. 2 (a) shows optical lithographic defined contacts and electrode pads, (b) shows the ebeam nanolithographic finger electrodes patterned between the contacts, (c) shows the photoresist protective window exposing the finger electrodes, but covering the rest of the sensor.
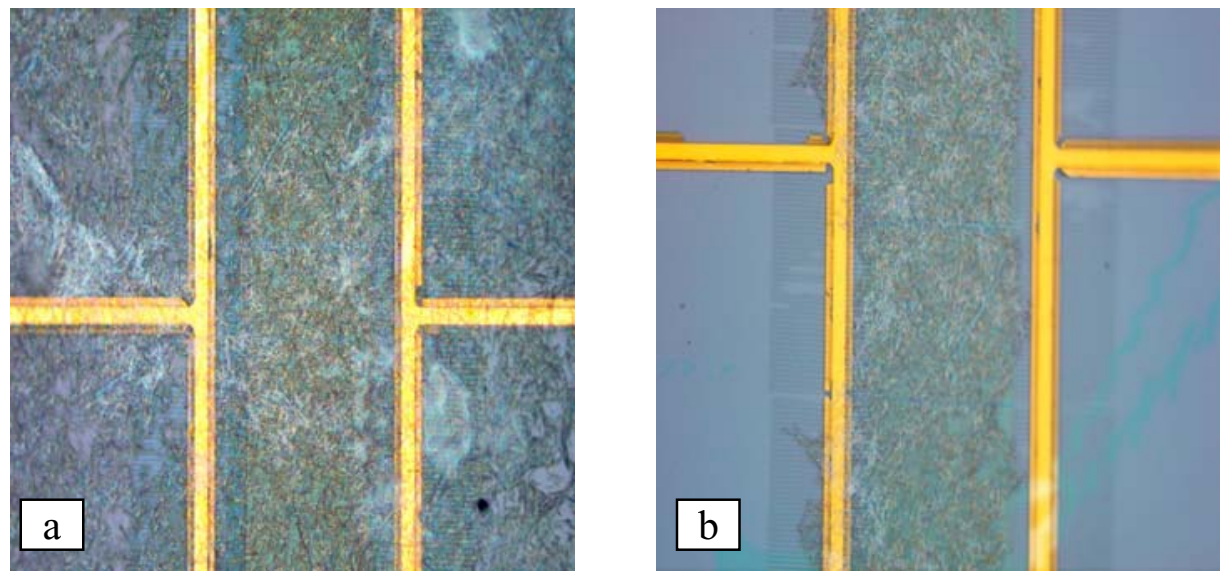

Fig. 3 (a) shows the sensor after Langmuir-Blodgett deposition of $\mathrm{ZnO}$ nanowires in single dense layer, (b) shows the sensor after removal of the protective window. 